\title{
ロバスト適応バックステッピングによる磁気浮上システムの制御*
}

\author{
水本 郁 朗*1, 大石 晋也年, 公文 誠*1 \\ 岩井善太*3, 神澤龍市*4
}

\section{Robust Adaptive Backstepping Control of a Magnetic Levitation System}

\author{
Ikuro MIZUMOTO*5, Shinya OHISHI, Makoto KUMON, \\ Zenta IWAI and Ryuichi KOZAWA \\ ${ }^{* 5}$ Graduate School of Science and Technology, Kumamoto University, \\ 2-39-1 Kurokami, Kumamoto-shi, Kumamoto, 860-8555 Japan
}

\begin{abstract}
It is very important to confirm the robust performance of the existing control scheme through experiments because several problems which might not satisfy the assumptions imposed on the controlled system are in hiding in practical applications. In this paper, we verify the effectiveness of the proposed robust adaptive backstepping control through experiment of a magnetic levitation system with uncertain nonlinearities.
\end{abstract}

Key Words: Robust Adaptive Control, Nonlinear Systems, Magnetic Levitation System, Backstepping

\section{1. 緒言}

実在するシステムの多くは何らかの非線形特性や不 確かさを有している. このようなことから, '90 年代の 初めより, 不確かな非線形に対する適応制御手法が意欲 的に研究されている(1) (5). 中でも適応バックステッピ ング手法は, 対象システムが'Parametric feedback form' に変換可能であれば, これまで必要とされていたマッ チング条件や Lipschitz 条件を満足しないより広範な 非線形システムに対して適用可能であった ${ }^{(5)}$. しかし, この手法の適用も不確かさが既知関数と未知定数との 線形和で表されるパラメトリックな不確かさを持つシ ステムに限定されていた.このようなことから, ノン パラメトリックな不確かさを持つ非線形システムに対 する適応制御手法がいくつか提案されている. 特に, 文献 ${ }^{(6)}(7)$ の手法は, 入力項にもノンパラメトリックな 不確かさを持つ非線形システムを対象としており,よ り一般的な非線形システムに対してもロバストな適応 制御が設計できる手法である. また，それらの有用性 は, 非線形性の強い完全混合タンク反応器 (CSTR) や 磁気浮上系のモデルを用いた数值シミュレーションに

\footnotetext{
* 原稿受付 2007 年 1 月 22 日.

*1 正員, 熊本大学大学院自然科学研究科(画860-8555 熊本市 黑髪 2-39-1)

*2 学生員, 熊本大学大学院自然科学研究科.

*3 正員, フェロー, 熊本大学大学院自然科学研究科.

*4 正員, 熊本大学工学部.

E-mail : ikuro@gpo.kumamoto-u.ac.jp
}

より検証されている、しかし，実際に制御手法を実シ ステムへ適用しようとする場合, 想定されていないさ まざまな要因により, 数值シミュレーションによる検 証では表れない問題に直面することがある.このよう なことから，提案された制御手法の有用性・実用性を 実機のシステムで検証し, 実用上有効な改善手法を示 すことは制御手法の実用化を図る上で重要なことであ る. 本論文では, 上述の不確かな非線形システム, 特 に入力項にもハンパラメトリックな不確かさを持つ非 線形システムにも有効な手法として著者らにより提案 されているハイゲインフィードバック型ロバスト適応 バックステッピング手法の実用性を一入出力磁気浮上 システムに対する実験により検証する. 本実験では, 文献 ${ }^{(6)}$ で示されているデッドゾーン法の適応調整則と $\sigma$ 修正法により制御系を構成した場合 $(\sigma$ 修正法によ る制御系の構成法および安定性解析は本論文中で新た に行なう), および PID 制御による場合について比較 検証し, 適用した適応制御手法の実際のシステムへの 応用に関する問題点を明らかにする. さらに, 明らか にされた問題点を考慮し, ノイズの影響やセットポイ ントが変化する場合などを想定したフィードバックゲ インの下限切り替え型 $\sigma$ 修正調整則による改善を試 みた結果を示す.ここで提案する下限切り替え型 $\sigma$ 修 正調整則による改善は, ハイゲインフィードバック型 ロバスト適応バックステッピング手法と $\sigma$ 修正法の特 性を生かしたものであり, 実際の応用において十分有 


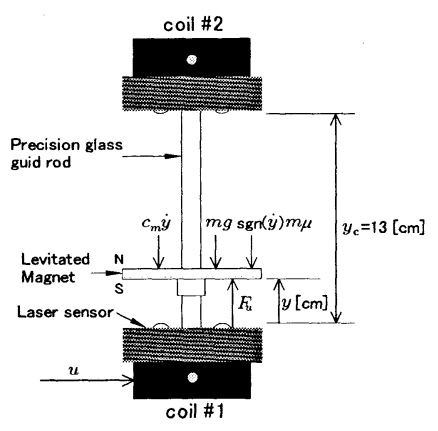

Fig. 1 Schematic diagram of magnetic levitation system

効な手法と考えられる.

\section{2. 装置の概 要}

今回使用した磁気浮上装置の概要を図 1 に示す。装 置には上部と下部にドライブコイルがあるが, 本稿で は下部のコイルのみを用いて実験を行う. 磁石がコイ ル間でグラスロッドにより支持され，上下方向のみの 運動に拘束されている. コイルにはアンプが取り付け られ，入力はアンプに対する入力電圧であり，それに よりコイルに直流電流が流れ, 磁界が発生する. 正の 電圧をかけると磁石に反発力が働き, 負の電圧をかけ ると吸引力が働く. 出力は下部にあるレーザ変位計に より磁石の高さを計測する. また, 装置には $\pm 3[\mathrm{~V}] よ$ りも大きな電圧が連続で加わらないようにする必要が ある(8).

3. 磁気浮上システムのモデル化

今回使用する装置での磁石の上下運動の運動方程式 は, 次式で記述される(8).

$$
\begin{gathered}
m \ddot{x}_{1}=F_{u}-c_{m} \dot{x}_{1}-m g \\
F_{u}=\frac{u}{a(y+b)^{N}}
\end{gathered}
$$

ここで, $x_{1}$ : 磁石の位置 $[\mathrm{m}], m=0.12$ : 磁石の質量 $[\mathrm{kg}], c_{m}$ : 粘性係数 $(=m \beta), g$ : 重力加速度 $\left[\mathrm{m} / \mathrm{s}^{2}\right], F_{u}$ : アクチュエータにより発生する磁力 $[\mathrm{N}], u$ : 入力電圧 [V] である. また $a, b, N$ は未知定数であり, 文献 ${ }^{(8)}$ によると $3<N<4.5$ とされている. ここではさらに, 中央に挿入されたグラスロッドと磁石との摩擦の影響 も考慮し，

$$
m \ddot{x}_{1}=F_{u}-c_{m} \dot{x}_{1}-m g-\operatorname{sgn}\left(\dot{x}_{1}\right) m \mu
$$

とする. 後述の適応バックステッピング手法では $m$, $\beta, g, a, b, N$ が未知として制御系が構成できる.
ここで, $x_{1}=10^{-2} y[\mathrm{~m}]$ であり, $x_{2}=\dot{x}_{1}[\mathrm{~m} / \mathrm{s}]$ とおけ ば, 式(3)は,

$$
\left\{\begin{array}{l}
\dot{x}_{1}=x_{2} \\
\dot{x}_{2}=f_{2}\left(x_{2}\right)+g_{2}\left(x_{1}\right) u \\
y=c x_{1}
\end{array}\right.
$$

と表すことができる.ここに,

$$
\begin{gathered}
f_{2}\left(x_{2}\right)=-\beta x_{2}-g-\operatorname{sgn}\left(x_{2}\right) \mu \\
g_{2}\left(x_{1}\right)=\frac{1}{a m\left(c x_{1}+b\right)^{N}} \\
c=10^{2}
\end{gathered}
$$

である.

\section{4. 適応バックステッピング制御系設計}

システム (4)のように表される二次の一入出力非線 形系に対し, 文献 ${ }^{(6)}$ をとにして構成した二つの適応 制御系について述べる.

初めにシステム (4) に対し, 以下の仮定をおく. [仮定]

(1) 未知非線形関数 $f_{2}\left(x_{2}\right)$ に対し,

$$
\left|f_{2}\right| \leq f_{02}\left|f_{M 2}\left(x_{2}\right)\right|+f_{12}
$$

なる関係を満足する未知正定数 $f_{02}, f_{12}$ および滑らか な既知関数 $f_{M 2}\left(x_{2}\right)$ が存在する.

(2) 末知非線形関数 $g_{2}\left(x_{1}\right)$ に対し,

$$
0<g_{m 2} \leq g_{2}\left(x_{2}\right)
$$

なる関係を満足する未知正定数 $g_{m 2}$ が存在する.

これらの仮定に対し, 対象とする磁気浮上システム では, 式(5)より

$$
f_{02}=\beta, f_{M 2}=x_{2}, f_{12}=g+\mu
$$

とおくことにより，仮定 (1) を満足する．また，仮定 (2) も式(6) と $0 \leq x_{1} \leq y_{c}=0.13[\mathrm{~m}]$ であることより満 足する.

このとき制御目的は, 出力 $y$ が与えられた規範信号 $y_{r}$ に追従する, すなわち微小正定数 $\delta$ に対し,

$$
\lim _{t \rightarrow \infty}\left|y-y_{r}\right| \leq \delta
$$

を満足する適応制御器を設計することである.ここに, $y_{r}$ は以下の条件を満足する信号である.

$$
\left\{\begin{array}{l}
\left|y_{r}(t)\right| \leq d_{0} \\
\left|\dot{y}_{r}(t)\right| \leq d_{1}
\end{array} \quad \forall t \in(0, \infty)\right.
$$

$d_{0}, d_{1}$ はある正定数である. 
4 .1 デッドゾーンによる制御系構成文献 もとに, 規範出力 $y_{r}[\mathrm{~cm}]$ に対し, 制御入力 $u=u_{d}$ を 以下のように設計する. ただし, 文献 ${ }^{(6)}$ の制御系構成 での最終ステップにおける積分項を一定值 $c_{d 2}$ として いる.これは, 速度のノイズの影響を考慮したためで ある. また, 文献 ${ }^{(6)}$ での正定值 $\gamma_{n P}$ を $\gamma_{d 21} \sim \gamma_{d 24}$ に分 割することで, 細かな調節を可能にしている.

$$
\begin{aligned}
& u_{d}=-\hat{k}_{d 2} z_{d 2} \\
& \hat{k}_{d 2}=c_{d 2}+\hat{k}_{d 2 P}, \quad c_{d 2}>0 \\
& \hat{k}_{d 2 P}=\gamma_{d 21} f_{M 2}^{2}+\gamma_{d 22} \hat{k}_{d 1}^{2} x_{2}^{2}+\gamma_{d 23} \hat{k}_{d 1}^{2}+\gamma_{d 24} z_{d 1}^{6} \\
& \gamma_{d 21}, \gamma_{d 22}, \gamma_{d 23}, \gamma_{d 24}>0 \text { (15) } \\
& \alpha_{d 1}=-\hat{k}_{d 1} z_{d 1} \\
& \dot{\hat{k}}_{d 1}=\gamma_{d 1} D\left(z_{d 1}\right) z_{d 1}^{2}, \hat{k}_{d 1}(0) \geq 0, \gamma_{d 1}>0 \\
& D\left(z_{d 1}\right)=\left\{\begin{array}{lll}
0 & \text { for } & \left|z_{d 1}\right| \leq \delta_{z} \\
1 & \text { for } & \left|z_{d 1}\right|>\delta_{z}
\end{array}\right.
\end{aligned}
$$

ここに, $z_{d 2}=10^{2} x_{2}-\alpha_{d 1}, z_{d 1}=y-y_{r}=10^{2} x_{1}-y_{r}$ で あり, $f_{M 2}$ は $f_{M 2}=x_{2}$ と与えている. また, $c_{d 2}, \gamma_{d 21}$ $\sim \gamma_{d 24}, \gamma_{d 1}, \delta_{z}, \hat{k}_{d 1}(0)$ は設計パラメータである.

$4.2 \sigma$ 修正による制御系構成規範出力 $y_{r}[\mathrm{~cm}]$ に対し, 制御入力 $u=u_{\sigma}$ を次のように設計する. 具 体的な制御系の構成法手順および安定性解析は付録 $\mathrm{A}$ に示す.

$$
\begin{aligned}
u_{\sigma}= & -\hat{k}_{\sigma 2} z_{\sigma 2} \\
\hat{k}_{\sigma 2}= & c_{\sigma 2}+\hat{k}_{\sigma 2 P}, \quad c_{\sigma 2}>0 \\
\hat{k}_{\sigma 2 P}= & \gamma_{\sigma 21} f_{M 2}^{2}+\gamma_{\sigma 22} \hat{k}_{\sigma 1}^{2} x_{2}^{2}+\gamma_{\sigma 23} \hat{k}_{\sigma 1}^{2} \\
& +\gamma_{\sigma 24} z_{\sigma 1}^{2}\left(\gamma_{\sigma 1} z_{\sigma 1}^{2}-\sigma_{1} \hat{k}_{\sigma 1}\right)^{2} \\
& \gamma_{\sigma 21}, \gamma_{\sigma 22}, \gamma_{\sigma 23}, \gamma_{\sigma 24}>0 \\
\alpha_{\sigma 1}= & -\hat{k}_{\sigma 1} z_{\sigma 1} \\
\dot{\hat{k}}_{\sigma 1}= & \gamma_{\sigma 1} z_{\sigma 1}^{2}-\sigma_{1} \hat{k}_{\sigma 1}, \\
& \hat{k}_{\sigma 1}(0) \geq 0, \gamma_{\sigma 1}>0, \sigma_{1}>0
\end{aligned}
$$

ここに, $z_{\sigma 2}=10^{2} x_{2}-\alpha_{\sigma 1}, z_{\sigma 1}=y-y_{r}=10^{2} x_{1}-y_{r}$ であり, $f_{M 2}$ は $f_{M 2}=x_{2}$ と与えている. また, $c_{\sigma 2}$, $\gamma_{\sigma 21} \sim \gamma_{\sigma 24}, \gamma_{\sigma 1}, \hat{k}_{\sigma 1}(0)$ および $\sigma_{1}$ は設計パラメータ である.

\section{5. 実 験 結 果}

比較のため本論文では PID による制御実験も行った. PID 制御器は以下のように構成した.

$$
u_{P I D}=\left(K_{p}+\frac{K_{i}}{s}+K_{d} s\right)\left[y_{r}-y\right]
$$

何れの制御手法においても制御系構成において磁石の 速度: $x_{2}$ が必要である.これはセンサから得られる磁石

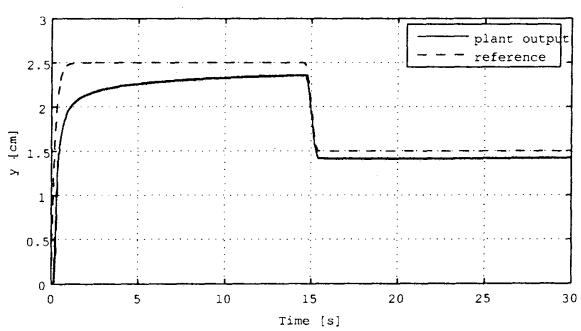

$c_{d 2}=0.1, \gamma_{d 21}=5 \times 10^{-5}, \gamma_{d 22}=5 \times 10^{-5}, \gamma_{d 23}=1 \times 10^{-8}, \gamma_{d 24}=$ $1 \times 10^{-1}, \gamma_{d 1}=4 \times 10, \delta_{z}=1 \times 10^{-2}(0.1 \mathrm{~mm}), \hat{k}_{d 1}(0)=20$

Fig. 2 dead-zone

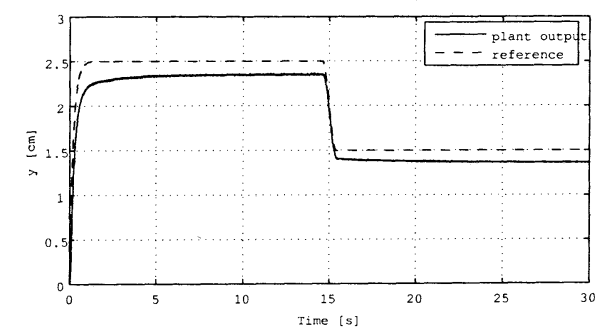

$c_{\sigma 2}=0.1, \gamma_{\sigma 21}=1 \times 10^{-4}, \gamma_{\sigma 22}=1 \times 10^{-4}, \gamma_{\sigma 23}=1 \times 10^{-8}, \gamma_{\sigma 24}=$ $1 \times 10^{-4}, \gamma_{\sigma 1}=1 \times 10^{2}, \sigma_{1}=5 \times 10^{-2}, \hat{k}_{\sigma 1}(0)=20$

Fig. $3 \quad \sigma$-modification

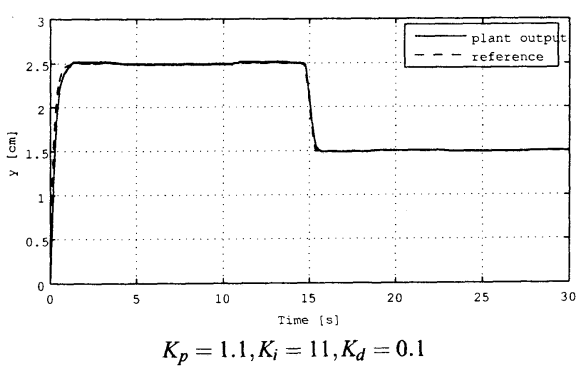

Fig. 4 PID

の位置: $x_{1}$ を微分することによって得ることにする. た だし，センサから得られる值には高周波ノイズが乗っ ているため, そのまま微分を行うことは望ましくない. そこで, ここでは, センサより得られる $x_{1}$ の值を, 試 行錯䛊により選んだローパスフィルタ $1 /(0.0008 s+1)^{2}$ に通して雑音を除去した後に差分をとった值を速度 $x_{2}$ として用いている．磁石の設定位置 $y_{r}$ は，条件 (12) を考慮して,

$$
\begin{aligned}
& y_{r}=\left\{\begin{array}{ccc}
\frac{5}{s+5}[2.5] & \text { for } & 0 \leq t<15-\frac{5}{12} \\
r & \text { for } & 15-\frac{5}{12} \leq t<15+\frac{5}{12} \\
1.5 & \text { for } & 15+\frac{5}{12} \leq t
\end{array}\right. \\
& r=-0.5 \sin \frac{2 \pi}{T} t+2, T=\frac{5}{3}
\end{aligned}
$$

とし，サンプリング間隔は $0.5[\mathrm{~ms}]$ とした. それぞれ の制御手法による実験結果を図 2〜図 4 に示す．PID 


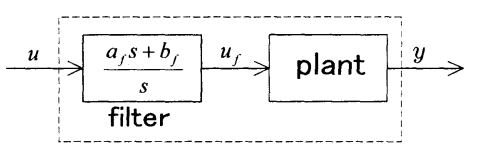

Fig. 5 introduction of filter

制御手法では，様々な規範信号に対して良好であった パラメータを用いている.

PID 制御手法では良好な結果が得られているが, 適 応バックステッピング法においては, 満足のいくような 結果が得られていないことが分かる.これは, 適応制 御手法においては追従䛊差が小さくなるようにフィー ドバックゲインを自動調整するが,ノイズの影響や入 力制限により適応ゲイン $\gamma_{d 1}, \gamma_{\sigma 1}$ を大きく設定できず, さらにデッドゾーン $\delta_{z}$ や 修正項を大きく設定しな ければならないためである.

6. 適応バックステッピング制御手法の改善

デッドゾーンによるバックステッピング制御では $\delta_{z}$ を大きく設定しても, 外力が加わったときなどにはゲ インが上昇し, いずれ装置の入力が破綻してしまう可 能性があるため, 今回の実験装置には適さないと判断 し， $\sigma$ 修正による制御手法でさらなる改善を試みた.

図 3 によると, 入力制限にかからないように選んだ 設計パラメータでは, 定常偏差が残ることが分かる. そこで, ここでは積分項を加えることで定常偏差を取 り除くことを考える. すなわち, 式(19)で得られる入 力 $u_{\sigma}$ に対し, 次のフィルタを導入した (図 5).

$$
\frac{a_{f} s+b_{f}}{s},\left(a_{f}, b_{f}: \text { 任意正定数 }\right)
$$

このフィルタ (26)の導入により, システムの次数が一 次増加したことがわかる. そこで次に, システム (4) にフィルタ (26) を導入した系について考察する.

フィルタ (26) は次の状態方程式で表される.

$$
\left\{\begin{array}{l}
\dot{x}_{3}=b_{f} u \\
u_{f}=x_{3}+a_{f} u
\end{array}\right.
$$

いま，(4) と (27) を併合した系を考えると，

$$
\left\{\begin{array}{l}
\dot{x}_{1}=x_{2} \\
\dot{x}_{2}=f_{2}\left(x_{2}\right)+g_{2}\left(x_{1}\right)\left(x_{3}+a_{f} u\right) \\
\dot{x}_{3}=b_{f} u \\
y=c x_{1}
\end{array}\right.
$$

となる.ここで, 次の変数変換を行う.

$$
\left\{\begin{array}{l}
\xi_{1}=x_{1} \\
\xi_{2}=x_{2} \\
\xi_{3}=x_{2}-\frac{a_{f}}{b_{f}} g_{2}\left(x_{1}\right) x_{3}
\end{array}\right.
$$

$\xi_{2}, \xi_{3}$ の微分は式(28)より,

$$
\begin{aligned}
\dot{\xi}_{2}= & f_{2}\left(x_{2}\right)+g_{2}\left(x_{1}\right)\left[\frac{1}{g_{2}\left(x_{1}\right)} \frac{b_{f}}{a_{f}}\left(\xi_{2}-\xi_{3}\right)+a_{f} u\right] \\
= & f_{2}\left(x_{2}\right)+\frac{b_{f}}{a_{f}}\left(\xi_{2}-\xi_{3}\right)+a_{f} g_{2}\left(x_{1}\right) u \\
\dot{\xi}_{3}= & f_{2}\left(x_{2}\right)+g_{2}\left(x_{1}\right)\left(x_{3}+a_{f} u\right)-\frac{a_{f}}{b_{f}} \dot{g}_{2}\left(x_{1}\right) x_{3} \\
& -\frac{a_{f}}{b_{f}} g_{2}\left(x_{1}\right) b_{f} u \\
= & f_{2}\left(x_{2}\right)+g_{2}\left(x_{1}\right) x_{3}-\frac{a_{f}}{b_{f}} \dot{g}_{2}\left(x_{1}\right) x_{3} \\
= & -\left[\frac{b_{f}}{a_{f}}-\frac{\dot{g}_{2}\left(x_{1}\right)}{g_{2}\left(x_{1}\right)}\right] \xi_{3}+\left[\frac{b_{f}}{a_{f}}-\frac{\dot{g}_{2}\left(x_{1}\right)}{g_{2}\left(x_{1}\right)}\right] \xi_{2} \\
& +f_{2}\left(x_{2}\right)
\end{aligned}
$$

となることから, 変数変換を行った系は次式で表さ れる.

$$
\left\{\begin{aligned}
\dot{\xi}_{1}= & \xi_{2} \\
\dot{\xi}_{2}= & f_{2}\left(x_{2}\right)+\frac{b_{f}}{a_{f}}\left(\xi_{2}-\xi_{3}\right)+a_{f} g_{2}\left(x_{1}\right) u \\
\dot{\xi}_{3}= & -\left[\frac{b_{f}}{a_{f}}-\frac{\dot{g}_{2}\left(x_{1}\right)}{g_{2}\left(x_{1}\right)}\right] \xi_{3}+\left[\frac{b_{f}}{a_{f}}-\frac{\dot{g}_{2}\left(x_{1}\right)}{g_{2}\left(x_{1}\right)}\right] \xi_{2} \\
& +f_{2}\left(x_{2}\right)
\end{aligned}\right.
$$

いま, 式(6)より,

$$
\dot{g}_{2}\left(x_{1}\right)=-\frac{N c \dot{x}_{1}}{a m\left(c x_{1}+b\right)^{N+1}}=-\frac{N c x_{2}}{a m\left(c x_{1}+b\right)^{N+1}}
$$

であるので,

$$
\begin{aligned}
\dot{\xi}_{3}= & -\left(\frac{b_{f}}{a_{f}}+\frac{N c \xi_{2}}{c x_{1}+b}\right) \xi_{3} \\
& +\left(\frac{b_{f}}{a_{f}}+\frac{N c \xi_{2}}{c x_{1}+b}\right) \xi_{2}+f_{2}\left(x_{2}\right)
\end{aligned}
$$

となる.

よって, 式(32)のゼロダイナミクスは, 式 (34)に $\xi_{1}=\xi_{2}=0$ を代入して

$$
\dot{\xi}_{3}=-\frac{b_{f}}{a_{f}} \xi_{3}-g
$$

を得る. $g$ は重力加速度 (定数外乱) であることから, $g \equiv 0$ としたゼロダイナミクスは安定である. すなわ ち, フィルタを含むシステム (32) は相対次数 2 の安定 なゼロダイナミクスを有するシステムであることが分 かる. この場合, (19)で構成した制御器をそのまま用 いることができる. すなわち, プロパなフィルタ (26) を導入することにより, システム全体の次数は増加す るが, 相対次数は変化しないため, 制御器を再設計す ることなく, 相対次数 2 のシステムに対して構成した 制御器 (19) をそのまま用いることができる. なお, 構 


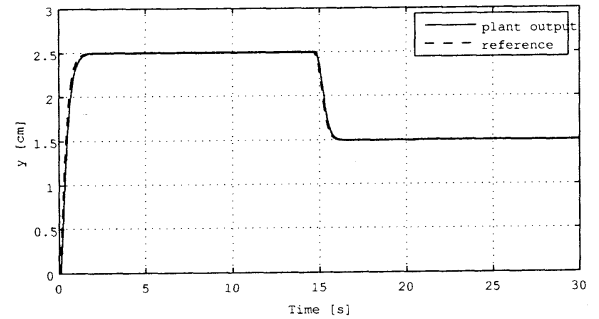

$c_{\sigma 2}=0.1, \gamma_{\sigma 21}=1 \times 10^{-4}, \gamma_{\sigma 22}=1 \times 10^{-4}, \gamma_{\sigma 23}=1 \times 10^{-8}, \gamma_{\sigma 24}=$ $1 \times 10^{-6}, \gamma_{\sigma 1}=1 \times 10^{2}, \sigma_{1}=1, \hat{k}_{\sigma 1}(0)=20, a_{f}=1, b_{f}=10$

Fig. $6 \quad \sigma$-modification-2

成された制御系（安定なゼロダイナミクスを持つシス テムに対する制御系) の安定性は，付録 A および文献 (9) と同様の手順で示すことができる.

このフィルタを導入した場合, 定常偏差が隇少する が，それとともに $\sigma$ 修正により推定ゲイン $\hat{k}_{\sigma 1}$ も滅少 し, 即応性が悪くなる恐れがある. そこで, 即応性の 悪化を防ぐために $\sigma$ 修正 $(23)$ を

$$
\begin{aligned}
& \dot{k}_{\sigma 1 \text { raw }}=\gamma_{\sigma 1} z_{\sigma 1}^{2}-\sigma_{1} \hat{k}_{\sigma 1} \\
& \begin{cases}\hat{k}_{\sigma 1}=\hat{k}_{\sigma 1 \text { raw }} \text { if } \hat{k}_{\sigma 1 \text { raw }}>\hat{k}_{\sigma 1 \text { low }}, \hat{k}_{\sigma 1 \text { low }}>0 \\
\hat{k}_{\sigma 1}=\hat{k}_{\sigma \text { llow }} \text { if } \hat{k}_{\sigma 1 \text { raw }} \leq \hat{k}_{\sigma 1 \text { low }}\end{cases}
\end{aligned}
$$

と改良することで $\hat{k}_{\sigma 1}$ の下限值を設定する. このとき の安定性も付録 A と同様にして保証される。

注意: 一般には, 適応制御手法で推定パラメータの推 定範囲を限定するためには, そのパラメータの存在範 囲が既知でなければ, 安定性は保証されない. しかし, 提案手法では，(37)において下限 $\hat{k}_{\text {ollow }}>0$ をどのよ うな值に設定してもシステムの安定性は保証される.

実験結果を図 6 に示す.ここでは, $\hat{k}_{\sigma 1}$ の下限值を 初期値 $\hat{k}_{\sigma 1}(0)$ と同様に $\hat{k}_{\sigma 1 \text { low }}=20$ としている. 提案 した改善手法により, 良好な結果を得ることができた。

\section{7. ロバスト性の検証}

次に, ロバスト性の検証の実験を行なった. 最初に 磁石に $200[\mathrm{~g}]$ のおもりを付加した状態で浮かせ, 途 中 3 秒付近でおもりを取り除き, 6 秒付近で再びおも りを付加する. 設計パラメータは先の実験と同じ值を 用いた。

磁石の設定位置 $y_{r}$ は,

$$
y_{r}=\frac{5}{s+5}[1]
$$

とした. 提案手法と PID 制御による実験結果をそれぞ れ図 7, 図 8 に示す. 図 7, 図 8 から提案手法の方が おもりの負荷の変動に対して, 振幅が小さく, 良好な 結果が得られていることが分かる. なお, 図 7 , 図 8

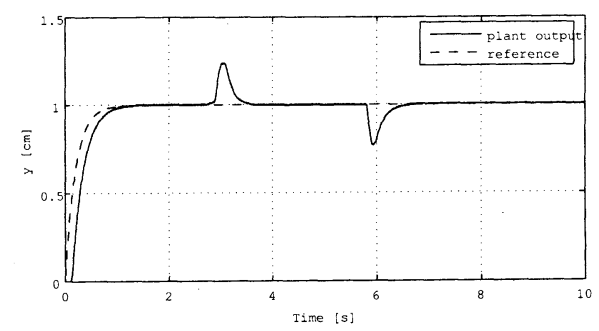

$c_{\sigma 2}=0.1, \gamma_{\sigma 21}=1 \times 10^{-4}, \gamma_{\sigma 22}=1 \times 10^{-4}, \gamma_{\sigma 23}=1 \times 10^{-8}, \gamma_{\sigma 24}=$ $1 \times 10^{-6}, \gamma_{\sigma 1}=1 \times 10^{2}, \sigma_{1}=1, \hat{k}_{\sigma 1}(0)=20, a_{f}=1, b_{f}=10$

Fig. $7 \sigma$-modification-2 〈experiment2〉

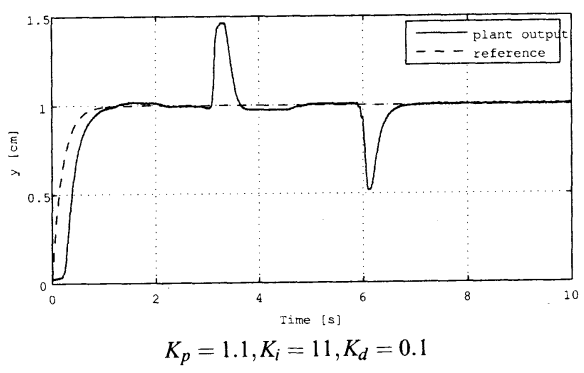

Fig. 8 PID 〈experiment2〉

に見られる制御開始初期の応答の遅れは，おもりを付 加したことによる磁石を支持するガイド棒の静止摩擦 力の増加等によるものと考えられる.

\section{8. 結言}

本報告では, 磁気浮上装置に対し適応バックステッ ピング法による制御系の構成を示し，その実用性につ いて検証した. 本実験では, PID 制御でも比較的良好 な結果が得られたが,フフルタを導入した $\sigma$ 修正を用 いた適応バックステッピング制御では,ささらに良好な 結果を得ることができた. また，おもりの負荷による ロバスト性についても検証した.

\section{文献}

(1) Polycarpou, M. M. and P. A. Ioannou, A robust adaptive nonlinear control design, Automatica, Vol.32, No.3, (1996) pp.423-427.

(2) Yao, B. and M. Tomizuka, Adaptive robust control of SISO nonlinear systems in a semi-strict feedback form, Automatica, Vol.33, No.5, (1997) pp.893-900.

(3) Jiang, Z-P. and L. Praly, Design of robust adaptive controller for nonlinear systems with dynamic uncertainties, Automatica, Vol.34, No.7 (1998) pp.825-840.

(4) Arslan, G. and T. Basar, Robust output feedback control of strict-feedback systems with unknown nonlinearities, Proc. of the 38th IEEE Conference of Decision and Control, Phocnix, Arizona (1999) pp.4748-4753

(5) Krstic, M. et al., Nonlinear and Adaptive Control Design, John Wiley \& Suns, NY (1995) 
(6) Mizumoto,I. et al., Robust Adaptive Backstepping Control Based on High-Gain Feedback and Its Application to a CSTR Control,Transactions of the Japan Society of Mechanical Engineers, Series C, Vol.69, No.686 (2003), pp.2667-2674

(7) Mizumoto,I. et al., Robust Adaptive Control for StrictFeedback Nonlinear Systems,Asian Journal of Control, Vol.7, No.3 (2005), pp.231-243

(8) Thomas R. Parks, Manual For Model 730 Magnetic Levitation System (Instractor's Edition)

(9) Mizumoto,I. et al., High Gain Adaptive Output Feedback Control for Time-Varying Nonlinear Systems with Higher Order Relative Degree,Transactions of the Society of Instrument and Control Engineers, Vol.40, No.10 (2004), pp.1014-1023.

(10) Michino,R. et al., Robust High Gain Adaptive Output Feedback Control for Nonlinear Systems with Uncertain Nonlinearities in Control Input Term,International Journal of Control,Automation, and Systems, Vol.1, No.1 (2003), pp.19-27.

\section{付録 A: $\sigma$ 修正法による制御系構成法}

ここでは，適応則に $\sigma$ 修正を用いた制御系構成法を 示す.

ステップ 1 :

規範出力 $y_{r}$ に対して, 誤差変数 $z_{\sigma 1}=y-y_{r}=c x_{1}-y_{r}$ を考えると， $z_{\sigma 1}$-システムは，

$$
\dot{z}_{\sigma 1}=c \dot{x}_{1}-\dot{y}_{r}=c x_{2}-\dot{y}_{r}
$$

と表される. そこで, この $z_{\sigma 1}$-システム内の $x_{2}$ に対 する仮想入力 $\alpha_{\sigma 1}$ を次のように構成する.

$$
\begin{gathered}
\alpha_{\sigma 1}=-\hat{k}_{\sigma 1} z_{\sigma 1} \\
\dot{\hat{k}}_{\sigma 1}=\gamma_{\sigma 1} z_{\sigma 1}^{2}-\sigma_{1} \hat{k}_{\sigma 1}, \hat{k}_{\sigma 1}(0) \geq 0, \gamma_{\sigma 1}>0(\mathrm{~A}-2)
\end{gathered}
$$

ここで, 次の正定值関数

$$
V_{\sigma 1}=\frac{1}{2} z_{\sigma 1}^{2}+\frac{1}{2 \gamma_{\sigma 1}}\left(\hat{k}_{\sigma 1}-k_{\sigma 1}^{*}\right)^{2}
$$

を考える. ここに, $k_{\sigma 1}^{*}$ は $\hat{k}_{\sigma 1}$ に対する未知理想フィー ドバックゲインであり, 後に決定する. この正定值関 数 $V_{\sigma 1}$ の時間微分は, 次のようになる.

$$
\begin{aligned}
\dot{V}_{\sigma 1}= & z_{\sigma 1}\left(\alpha_{\sigma 1}+z_{\sigma 2}-\dot{y}_{r}\right)+\left(\hat{k}_{\sigma 1}-k_{\sigma 1}^{*}\right) z_{\sigma 1}^{2} \\
& -\frac{\sigma_{1}}{\gamma_{\sigma 1}}\left(\hat{k}_{\sigma 1}-k_{\sigma 1}^{*}\right) \hat{k}_{\sigma 1} \\
= & z_{\sigma 1}\left(\alpha_{\sigma 1}+z_{\sigma 2}-\dot{y}_{r}\right)+\left(\hat{k}_{\sigma 1}-k_{\sigma 1}^{*}\right) z_{\sigma 1}^{2} \\
& -\frac{\sigma_{1}}{\gamma_{\sigma 1}}\left[\left(\hat{k}_{\sigma 1}-k_{\sigma 1}^{*}\right)^{2}+\left(\hat{k}_{\sigma 1}-k_{\sigma 1}^{*}\right) k_{\sigma 1}^{*}\right](\mathrm{A}-6)
\end{aligned}
$$

ここに, $z_{\sigma 2}=c x_{2}-\alpha_{\sigma 1}$ である. 式(A-2), (A-3) で与 えた $\alpha_{\sigma 1}$ の構造から,

$$
\begin{aligned}
\dot{V}_{\sigma 1}= & z_{\sigma 1} z_{\sigma 2}-z_{\sigma 1} \dot{y}_{r}-k_{\sigma 1}^{*} z_{\sigma 1}^{2} \\
& -\frac{\sigma_{1}}{\gamma_{\sigma 1}}\left[\left(\hat{k}_{\sigma 1}-k_{\sigma 1}^{*}\right)^{2}+\left(\hat{k}_{\sigma 1}-k_{\sigma 1}^{*}\right) k_{\sigma 1}^{*}\right](\mathrm{A}-7)
\end{aligned}
$$

となる. よって, $\left|\dot{y}_{r}\right| \leq d_{1}$ であることより, $\dot{V}_{\sigma 1}$ は任 意正定数 $\rho_{\sigma 1}$ を用いて,

$$
\begin{aligned}
\dot{V}_{\sigma 1} \leq & -\left(k_{\sigma 1}^{*}-\rho_{\sigma 1}\right) z_{\sigma 1}^{2}-\rho_{\sigma 1} z_{\sigma 1}^{2}+d_{1}\left|z_{\sigma 1}\right| \\
& +z_{\sigma 1} z_{\sigma 2}-\frac{\sigma_{1}}{\gamma_{\sigma 1}}\left(\hat{k}_{\sigma 1}-k_{\sigma 1}^{*}\right)^{2} \\
& -\frac{\sigma_{1}}{\gamma_{\sigma 1}}\left(\hat{k}_{\sigma 1}-k_{\sigma 1}^{*}\right) k_{\sigma 1}^{*}
\end{aligned}
$$

と評価できる。また，

$$
\begin{gathered}
-\frac{\sigma_{1}}{2 \gamma_{\sigma 1}}\left(\hat{k}_{\sigma 1}-k_{\sigma 1}^{*}\right)^{2}-\frac{\sigma_{1}}{\gamma_{\sigma 1}}\left(\hat{k}_{\sigma 1}-k_{\sigma 1}^{*}\right) k_{\sigma 1}^{*} \leq \frac{\sigma_{1} k_{\sigma 1}^{*}{ }^{2}}{2 \gamma_{\sigma 1}} \\
-\rho_{\sigma 1} z_{\sigma 1}^{2}+d_{1}\left|z_{\sigma 1}\right| \leq \frac{d_{1}^{2}}{4 \rho_{\sigma 1}}
\end{gathered}
$$

となることを考慮すれば， $V_{\sigma 1}$ の時間微分は,

$$
\begin{gathered}
\dot{V}_{\sigma 1} \leq-\left(k_{\sigma 1}^{*}-\rho_{\sigma 1}\right) z_{\sigma 1}^{2}-\frac{\sigma_{1}}{2 \gamma_{\sigma 1}}\left(\hat{k}_{\sigma 1}-k_{\sigma 1}^{*}\right)^{2} \\
+z_{\sigma 1} z_{\sigma 2}+R_{\sigma 1} \\
R_{\sigma 1}=\frac{d_{1}^{2}}{4 \rho_{\sigma 1}}+\frac{\sigma_{1} k_{\sigma 1}^{*}{ }^{2}}{2 \gamma_{\sigma 1}}
\end{gathered}
$$

と評価できる.

ステップ 2:

式(A-11)より $z_{\sigma 2}=0$ となれば, 制御系は安定とな る. そこでここでは， $z_{\sigma 2}$-システムを考える.

$$
\begin{aligned}
& z_{\sigma 2} \text {-システムは } \\
& \begin{aligned}
\dot{z}_{\sigma 2} & =c \dot{x}_{2}-\dot{\alpha}_{\sigma 1} \\
& =c\left(f_{2}+g_{2} u\right)-\left(\frac{\partial \alpha_{\sigma 1}}{\partial x_{1}} x_{2}+\frac{\partial \alpha_{\sigma 1}}{\partial y_{r}} \dot{y}_{r}+\frac{\partial \alpha_{\sigma 1}}{\partial \hat{k}_{\sigma 1}} \dot{\hat{k}}_{\sigma 1}\right)
\end{aligned}
\end{aligned}
$$

と表される. そこで式(A-13)を考慮し， $z_{\sigma 2}$-システム 内の実入力 $u=u_{\sigma}$ を

$$
\begin{aligned}
& u_{\sigma}=-\hat{k}_{\sigma 2} z_{\sigma 2} \\
& \hat{k}_{\sigma 2}= c_{\sigma 2}+\hat{k}_{\sigma 2 P}, \quad c_{\sigma 2}>0 \\
& \hat{k}_{\sigma 2 P}= \gamma_{\sigma 21} f_{M 2}^{2}+\gamma_{\sigma 22}\left|\frac{\partial \alpha_{\sigma 1}}{\partial x_{1}}\right|^{2} x_{2}^{2}+\gamma_{\sigma 23}\left|\frac{\partial \alpha_{\sigma 1}}{\partial y_{r}}\right|^{2} \\
&+\gamma_{\sigma 24}\left|\frac{\partial \alpha_{\sigma 1}}{\partial \hat{k}_{\sigma 1}}\right|^{2}\left(\gamma_{\sigma 1} z_{\sigma 1}^{2}-\sigma_{1} \hat{k}_{\sigma 1}\right)^{2} \\
& \gamma_{\sigma 21}, \gamma_{\sigma 22}, \gamma_{\sigma 23}, \gamma_{\sigma 24}>0
\end{aligned}
$$

と構成する.

ここで, 次の正定值関数

$$
V_{\sigma}=V_{\sigma 1}+\frac{1}{2} z_{\sigma 2}^{2}
$$

を考える.この $V_{\sigma}$ の時間微分は

$$
\dot{V}_{\sigma}=\dot{V}_{\sigma 1}+z_{\sigma 2} \dot{z}_{\sigma 2}
$$




$$
\begin{aligned}
= & \dot{V}_{\sigma 1}+c f_{2} z_{\sigma 2}+c g_{2} z_{\sigma 2} u_{\sigma}-\frac{\partial \alpha_{\sigma 1}}{\partial x_{1}} x_{2} z_{\sigma 2} \\
& -\frac{\partial \alpha_{\sigma 1}}{\partial y_{r}} \dot{y}_{r} z_{\sigma 2}-\frac{\partial \alpha_{\sigma 1}}{\partial \hat{k}_{\sigma 1}}\left(\gamma_{\sigma 1} z_{\sigma 1}^{2}-\sigma_{1} \hat{k}_{\sigma 1}\right) z_{\sigma 2} \\
\leq & -\left(k_{\sigma 1}^{*}-\rho_{\sigma 1}\right) z_{\sigma 1}^{2}-\frac{\sigma_{1}}{2 \gamma_{\sigma 1}}\left(\hat{k}_{\sigma 1}-k_{\sigma 1}^{*}\right)^{2} \\
& +\left|z_{\sigma 1}\right|\left|z_{\sigma 2}\right|+R_{\sigma 1}+c f_{02}\left|f_{M 2}\right|\left|z_{\sigma 2}\right|+c f_{12}\left|z_{\sigma 2}\right| \\
& +c g_{2} z_{\sigma 2} u_{\sigma}+\left|\frac{\partial \alpha_{\sigma 1}}{\partial x_{1}}\right|\left|x_{2}\right|\left|z_{\sigma 2}\right|+d_{1}\left|\frac{\partial \alpha_{\sigma 1}}{\partial y_{r}}\right|\left|z_{\sigma 2}\right| \\
& +\left|\frac{\partial \alpha_{\sigma 1}}{\partial \hat{k}_{\sigma 1}}\right|\left|\gamma_{\sigma 1} z_{\sigma 1}^{2}-\sigma_{1} \hat{k}_{\sigma 1}\right|\left|z_{\sigma 2}\right|
\end{aligned}
$$

と評価でき，さらに仮定 (2) および入力 (19) を考慮す れば,

$$
\begin{aligned}
\dot{V}_{\sigma} \leq & -\left(k_{\sigma 1}^{*}-\rho_{\sigma 1}\right) z_{\sigma 1}^{2}-\frac{\sigma_{1}}{2 \gamma_{\sigma 1}}\left(\hat{k}_{\sigma 1}-k_{\sigma 1}^{*}\right)^{2} \\
& +\left|z_{\sigma 1}\right|\left|z_{\sigma 2}\right|+R_{\sigma 1}+c f_{02}\left|f_{M 2}\right|\left|z_{\sigma 2}\right|+c f_{12}\left|z_{\sigma 2}\right| \\
& -c g_{m 2} z_{\sigma 2}^{2}\left[c_{\sigma 2}+\gamma_{\sigma 21} f_{M 2}^{2}+\gamma_{\sigma 22}\left|\frac{\partial \alpha_{\sigma 1}}{\partial x_{1}}\right|^{2} x_{2}^{2}\right. \\
& \left.+\gamma_{\sigma 23}\left|\frac{\partial \alpha_{\sigma 1}}{\partial y_{r}}\right|^{2}+\gamma_{\sigma 24}\left|\frac{\partial \alpha_{\sigma 1}}{\partial \hat{k}_{\sigma 1}}\right|^{2}\left(\gamma_{\sigma 1} z_{\sigma 1}^{2}-\sigma_{1} \hat{k}_{\sigma 1}\right)^{2}\right] \\
& +\left|\frac{\partial \alpha_{\sigma 1}}{\partial x_{1}}\right|\left|x_{2}\right|\left|z_{\sigma 2}\right|+d_{1}\left|\frac{\partial \alpha_{\sigma 1}}{\partial y_{r}}\right|\left|z_{\sigma 2}\right| \\
& +\left|\frac{\partial \alpha_{\sigma 1}}{\partial \hat{k}_{\sigma 1}}\right|\left|\gamma_{\sigma 1} z_{\sigma 1}^{2}-\sigma_{1} \hat{k}_{\sigma 1}\right|\left|z_{\sigma 2}\right|
\end{aligned}
$$

となる.ここで,

$$
-c g_{m 2} \gamma_{\sigma 21} f_{M 2}^{2} z_{\sigma 2}^{2}+c f_{02}\left|f_{M 2}\right|\left|z_{\sigma 2}\right| \leq \frac{c f_{02}^{2}}{4 \gamma_{\sigma 21} g_{m 2}}
$$$$
-c g_{m 2} \gamma_{\sigma 22}\left|\frac{\partial \alpha_{\sigma 1}}{\partial x_{1}}\right|^{2} x_{2}^{2} z_{\sigma 2}^{2}+\left|\frac{\partial \alpha_{\sigma 1}}{\partial x_{1}}\right|\left|x_{2}\right|\left|z_{\sigma 2}\right|
$$

$$
\leq \frac{1}{4 c \gamma_{\sigma 22} g_{m 2}}
$$

$$
\begin{array}{r}
-c g_{m 2} \gamma_{\sigma 23}\left|\frac{\partial \alpha_{\sigma 1}}{\partial y_{r}}\right|^{2} z_{\sigma 2}^{2}+d_{1}\left|\frac{\partial \alpha_{\sigma 1}}{\partial y_{r}}\right|\left|z_{\sigma 2}\right| \\
\leq \frac{d_{1}^{2}}{4 c \gamma_{\sigma 23} g_{m 2}}
\end{array}
$$

$$
\begin{aligned}
& -c g_{m 2} \gamma_{\sigma 24}\left|\frac{\partial \alpha_{\sigma 1}}{\partial \hat{k}_{\sigma 1}}\right|^{2}\left(\gamma_{\sigma 1} z_{\sigma 1}^{2}-\sigma_{1} \hat{k}_{\sigma 1}\right)^{2} z_{\sigma 2}^{2} \\
& +\left|\frac{\partial \alpha_{\sigma 1}}{\partial \hat{k}_{\sigma 1}}\right|\left|\gamma_{\sigma 1} z_{\sigma 1}{ }^{2}-\sigma_{1} \hat{k}_{\sigma 1}\right|\left|z_{\sigma 2}\right| \leq \frac{1}{4 c \gamma_{\sigma 24} g_{m 2}}
\end{aligned}
$$

であること, また, 任意正定数 $\rho_{\sigma 2}, \rho_{\sigma 3}$ を用いて

$$
\begin{aligned}
& -\rho_{\sigma 2} z_{\sigma 2}^{2}+c f_{12}\left|z_{\sigma 2}\right| \leq \frac{c^{2} f_{12}^{2}}{4 \rho_{\sigma 2}} \\
& -\rho_{\sigma 3} z_{\sigma 2}^{2}+\left|z_{\sigma 1}\right|\left|z_{\sigma 2}\right| \leq \frac{z_{\sigma 1}^{2}}{4 \rho_{\sigma 3}}
\end{aligned}
$$

であることを考慮すれば, $V_{\sigma}$ の時間微分は,

$$
\dot{V}_{\sigma} \leq-\left(k_{\sigma 1}^{*}-\rho_{\sigma 1}-\frac{1}{4 \rho_{\sigma 3}}\right) z_{\sigma 1}^{2}
$$

$$
\begin{aligned}
& -\left(c g_{m 2} c_{\sigma 2}-\rho_{\sigma 2}-\rho_{\sigma 3}\right) z_{\sigma 2}^{2} \\
& -\frac{\sigma_{1}}{2 \gamma_{\sigma 1}}\left(\hat{k}_{\sigma 1}-k_{\sigma 1}^{*}\right)^{2}+R_{\sigma 2}
\end{aligned}
$$

と評価できる.ここに，

$$
\begin{aligned}
R_{\sigma 2}= & R_{\sigma 1}+\frac{c f_{02}^{2}}{4 \gamma_{\sigma 21} g_{m 2}}+\frac{1}{4 c \gamma_{\sigma 22} g_{m 2}}+\frac{d_{1}^{2}}{4 c \gamma_{\sigma 23} g_{m 2}} \\
& +\frac{1}{4 c \gamma_{\sigma 24} g_{m 2}}+\frac{c^{2} f_{12}^{2}}{4 \rho_{\sigma 2}}
\end{aligned}
$$

である. 最後に $\rho_{\sigma 2}=\rho_{\sigma 3}=c g_{m 2} c_{\sigma 2} / 4$ とおき, さら に $k_{\sigma 1}^{*}$ が

$$
\frac{1}{2} \leq k_{\sigma 1}^{*}-\rho_{\sigma 1}-\frac{1}{\operatorname{cg}_{m 2} c_{\sigma 2}}
$$

となるように設定すれば， $V_{\sigma}$ の時間微分は結局

$$
\begin{aligned}
& \dot{V}_{\sigma} \leq-\left(k_{\sigma 1}^{*}-\rho_{\sigma 1}-\frac{1}{c g_{m 2} c_{\sigma 2}}\right) z_{\sigma 1}^{2}-\frac{c g_{m 2} c_{\sigma 2}}{2} z_{\sigma 2}^{2} \\
&-\frac{\sigma_{1}}{2 \gamma_{\sigma 1}}\left(\hat{k}_{\sigma 1}-k_{\sigma 1}^{*}\right)^{2}+R_{\sigma 2} \\
& \leq-\alpha_{n} V_{\sigma}+R_{\sigma 2} \\
& \alpha_{n}= \min \left[2\left(k_{\sigma 1}^{*}-\rho_{\sigma 1}-\frac{1}{c g_{m 2} c_{\sigma 2}}\right)\right. \\
&\left., c g_{m 2} c_{\sigma 2}, \sigma_{1}\right]
\end{aligned}
$$

と評価できる.よって, 式(A-32)より, システムの 全信号は有界となることがわかる.ささら式 (A-32) から,

$$
\lim _{t \rightarrow \infty} V_{\sigma} \leq \frac{R_{\sigma 2}}{\alpha_{n}}
$$

が得られる, また $V_{\sigma}$ の定義より，

$$
z_{\sigma 1}^{2} \leq 2 V_{\sigma}
$$

であることから，結局

$$
\lim _{t \rightarrow \infty} z_{\sigma 1}^{2} \leq \frac{2 R_{\sigma 2}}{\alpha_{n}}
$$

と評価できる. すなわち, $\delta^{2} \geq 2 R_{\sigma 2} / \alpha_{n}$ となる $\delta$ に 対して, 制御目的 (11)を満足する. よって, 設計パラ メータ $\gamma_{\sigma 1}, c_{\sigma 2}, \gamma_{\sigma 21} \sim \gamma_{\sigma 24}$ を適切に設定することによ り, 任意の $\delta$ に対し, 制御目的 (11) を満足すること ができる(10).

上記に従って制御系を構成したとき, 次の定理が得 られる.

[定理]

仮定 (1)，(2)を満足するシステム (4)に対し，ステッ プ 1, ステップ 2 に従い制御入力 $u$ を式 (19) で構成す る. このとき, 構成された制御系内の全信号は有界で あり, さらに追従誤差は, 任意の与えられた正定数 $\delta$ に対し, 制御目的 (11)を満足する適切な設計パラメー タ $\gamma_{\sigma 1}, c_{\sigma 2}, \gamma_{\sigma 21} \sim \gamma_{\sigma 24}$ が必ず存在する. 